Anaesthesist 2009 $\cdot 58: 569-570$ DOI 10.1007/s00101-009-1567-3

Online publiziert: 20. Mai 2009

(c) Springer Medizin Verlag 2009

\author{
B. Heindl \\ Klinik für Anaesthesiologie, Klinikum der Universität München
}

\title{
Das familienfreundliche Krankenhaus
}

\section{Eine berufspolitische Herausforderung}

Im ersten Quartal 2009 gab die Bundesärztekammer die aktuellen Arztzahlen für das Jahr 2008 bekannt [1]. Nur 2848 Ärzte waren im September 2008 in Deutschland arbeitslos gemeldet. Im Vergleich zum Höhepunkt der Ärztearbeitslosigkeit im September 1997 entspricht dies einem Rückgang von 70\%. Die aktuelle Arbeitslosenquote von $0,9 \%$, bezogen auf alle berufstätigen Ärzte, kommt damit der Vollbeschäftigung im ärztlichen Dienst gleich. Parallel dazu sind im Jahr 20083065 ursprünglich in Deutschland tätige Ärzte ins Ausland abgewandert, mehr als je zuvor. Die Rekrutierung von ärztlichem Personal wird durch diese Situation für die Krankenhäuser immer schwieriger.

Die Analyse zeigt weiterhin, dass die „Feminisierung“ des ärztlichen Berufes anhält. Der Anteil der Ärztinnen an den Erstmeldungen bei den Ärztekammern erreichte 2008 einen neuen Höchststand von $57,9 \%$. Der Prozentsatz der Ärztinnen an der Gesamtzahl der berufstätigen Ärzte liegt aktuell bei $41,5 \%$. Anfang der 9oer Jahre entsprach diese Quote noch rund einem Drittel. Gerade für junge Ärztinnen stellt sich bei der Wahl ihres Berufsfeldes und -faches oft die Frage der Vereinbarkeit von Familie und Beruf. Dieser Punkt hat für die berufliche Entscheidung eine hohe Wertigkeit.

Von den nichtärztlich tätigen Ärzten waren 2008 10,6\% - das entspricht einer Zahl von über 10.000 - ausschließlich im Haushalt tätig oder befanden sich in Elternzeit. Für die Arbeitgeber birgt dies zumindest theoretisch - die Chance, auf eine große und häufig bereits berufser- fahrene Gruppe von potenziellen Bewerbern für ärztliche Stellen zurückgreifen zu können. Voraussetzung für die vermehrte Rückkehr von Ärzten aus dem reinen Familienengagement in das Berufsleben ist jedoch die Vereinbarkeit von Familie und Beruf.

Die Personalverantwortlichen der Krankenhäuser sind aufgerufen, die notwendigen Veränderungen herbeizuführen, um den Arbeitsplatz für Mitarbeiter mit Kindern attraktiv zu gestalten. Dafür steht ein ganzes Arsenal an Möglichkeiten zur Verfügung: z. B. Angebot von Teilzeitstellen, flexiblere Arbeitszeitmodelle, Kinderbetreuungsmöglichkeiten, „job sharing“. Solche Veränderungen erfordern kreatives Denken und Handeln von Personalverantwortlichen und manchmal auch Investitionen der Krankenhäuser. Dass sich ein solches Vorgehen letztendlich auch ökonomisch auszahlen kann, zeigt eindrucksvoll das Beispiel der Berufsgenossenschaftlichen Unfallklinik Murnau [2]. Diese Klinik bietet für Mitarbeiter die Betreuung ihrer Kinder an 365 Tagen im Jahr an. Von 5.30 Uhr am Morgen bis 21.30 Uhr am Abend ist die Einrichtung für Kinder im Alter von 8 Wochen bis zu 10 Jahren Ort der Betreuung. Die Berechnung der Wirtschaftlichkeit dieser Einrichtung im Jahr 2008 zeigte, dass sich eine positive Kosten-Nutzen-Differenz von etwa EUR 136.00o/Jahr ergibt. Kosteneinsparungen können vor allem über eine reduzierte Personalakquise realisiert werden, da Mitarbeiter mit Kindern durch das hervorragende Betreuungsangebot im Beruf gehalten werden. Wieder- 
beschaffungs-, Überbrückungs- und Wiedereingliederungskosten für Personal lassen sich nachhaltig reduzieren.

Im Beitrag von Heller u. Heller [3] werden die Probleme und Lösungsmöglichkeiten bei der Vereinbarkeit von Familie und Beruf differenziert aufgezeigt. Die Autoren bereichern das Thema mit ihren eigenen Erfahrungen in der Umsetzung familienfreundlicher Personalstrategien an der Klinik für Anästhesiologie der Universität Dresden, die sich bereits seit Jahren mit diesem Thema befasst. Ihrer Schlussfolgerung, dass eine familienfreundliche Familienpolitik für Krankenhäuser zukünftig ein wesentlicher Wettbewerbsvorteil bei der Rekrutierung von ärztlichem und pflegerischem Fachpersonal sein wird, kann ohne Einschränkung zugestimmt werden. Dieser berufspolitischen Herausforderung werden sich die Kliniken und Abteilungen für Anästhesiologie zukünftig verstärkt stellen müssen.

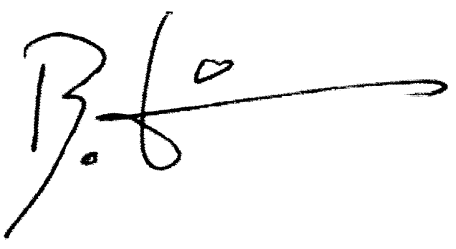

B. Heindl

\section{Korrespondenzadresse}

Prof. Dr. B. Heindl

Klinik für Anaesthesiologie,

Klinikum der Universität München, Nussbaumstraße 20, 80336 München

bernhard.heindl@med.uni-muenchen.de

Interessenkonflikt. Der korrespondierende Autor gibt an, dass kein Interessenkonflikt besteht.

\section{Literatur}

1. Kopetsch T (2009) Arztzahlenentwicklung: Hohe Abwanderung ins Ausland - sehr geringe Arbeitslosigkeit. Dtsch Arztebl 106:A-757
2. Dobner $P$ (2008) Rechnerische Darstellung der Kosten-Nutzen-Gegenüberstellung am Beispiel der Unfallklinik Murnau auf Basis der Studie Betriebswirtschaftliche Effekte familienfördernder Maßnahmen der Prognos AG. http://www.bgumurnau.de/Klinikdienste/Kindertagesstaette/index.php

3. Heller AR, Heller SC (2009) Die familienfreundliche Klinik - (wie) geht das? Anaesthesist 58:571-581

\section{(>) Exklusiv für Abonnenten :}

\section{Nutzen Sie das Online-Archiv von Der Anaesthesist}

Ihre Vorteile:

- Komfortable und schnelle Recherche nach Themen, Autoren, Suchbegriffen

— Ob unterwegs oder am eigenen PC: Zugriff überall und jederzeit

- Online First: Lesen Sie die aktuellsten Beiträge schon vor Erscheinen des gedruckten Heftes online

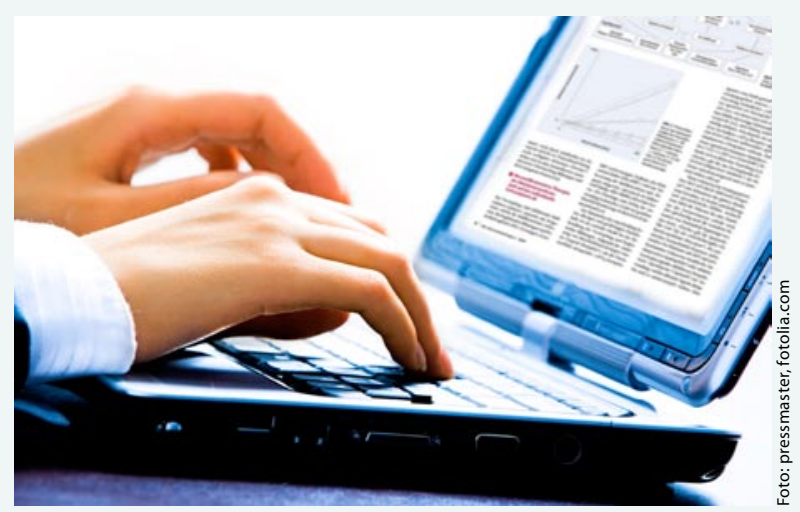

